\title{
Phase diagrams and universality classes of random antiferromagnetic spin ladders
}

\author{
J. A. Hoyos and E. Mirand A $^{*}$ \\ Instituto de Física Gleb Wataghin, Unicamp, Caixa Postal 6165, 13083-970. Campinas, SP, Brazil
}

(Dated: 29th October 2018)

\begin{abstract}
The random antiferromagnetic two-leg and zigzag spin-1/2 ladders are investigated using the real space renormalization group scheme and their complete phase diagrams are determined. We demonstrate that the first system belongs to the same universality class of the dimerized random spin-1/2 chain. The zigzag ladder, on the other hand, is in a random singlet phase at weak frustration and disorder. Otherwise, we give additional evidence that it belongs to the universality class of the random antiferromagnetic and ferromagnetic quantum spin chains, although the universal fixed point found in the latter system is never realized. We find, however, a new universal fixed point at intermediate disorder.
\end{abstract}

PACS numbers: $75.10 . J m, 75.10 . \mathrm{Nr}$

\section{INTRODUCTION}

One dimensional spin systems have been extensively studied over the last several years and a fairly deep understanding of their possible phases and corresponding physical behavior has emerged. Although actual realizations are restricted to systems with a high degree of anisotropy, one of the main motivations for these studies is the possibility of cataloging their universality classes. This is specially tempting in the case of disordered systems, since specially powerful methods can be employed in one dimension, which are able to expose not only average values but full distribution functions. One of the most studied random one-dimensional spin systems is the random antiferromagnetic (AF) spin- $1 / 2$ chain ${ }^{1.2}$ Making use of the real space renormalization group (RSRG) method of Ma, Dasgupta and $\mathrm{Hu}, \stackrel{1}{=}$ it has been shown that, for any amount of uncorrelated disorder, 3 the lowenergy physics of this system is governed by an infinite randomness fixed point ${ }^{2}$ The approach to this fixed point is characterized by the formation at decreasing energy scales of random singlets between widely separated spins. In this random singlet (RS) phase several physical properties are universal and known ${ }^{2}$ For example, the spin susceptibility $\chi \sim 1 / T \log ^{2} T$, and the spinspin correlation function $C_{i j}=\left\langle\mathbf{S}_{i} \cdot \mathbf{S}_{j}\right\rangle$ is such that its mean value $\overline{C_{i j}} \sim(-1)^{i-j} /|i-j|^{2}$, while the typical one $\left|C_{i j}\right|_{\text {typ }} \sim \exp (-\sqrt{|i-j|})$.

Other random systems have also been analyzed by these methods, among which two are of special interest to us. One is the random dimerized AF chain with different distributions for odd and even links. ${ }^{4}$ The presence of a gap in the clean version of this system provides protection against the introduction of disorder. Therefore, for weak disorder, the system retains a gap (or a pseudogap) and a spin susceptibility which decreases to zero with decreasing temperature. For strong enough disorder, however, the pseudogap is destroyed and the susceptibility diverges as a power law. The power law exponent is non-universal and varies continuously with the disorder strength, characterizing a Griffiths phase.
The other system of interest is the random chain with both $\mathrm{AF}$ and ferromagnetic (FM) interactions ${ }^{5.6}$ It has been shown, using a generalization of the RSRG procedure, that the low-energy behavior of this system is governed by the formation of long clusters with large total spins. For weak disorder, this large spin (LS) phase is universal. The magnetic susceptibility diverges like a Curie law, and the specific heat vanishes like $T^{1 / z}|\ln T|$, with $1 / z \approx 0.44$. However, for strong disorder, the LS phase is no longer universal: although the magnetic susceptibility is still Curie-like, the specific heat exponent $z$ varies continuously with disorder.

More recently, following the discovery of spin ladder materials,,$\frac{7}{\underline{T}}$ attention has been drawn to coupled pairs of spin chains. The random two-leg and zigzag ladders are the most studied of them ${ }^{8.9 .10}$ The two-leg ladder is known to possess two phases: ${ }^{8.9}$ a gapped phase, with vanishing spin susceptibility $\chi(T)$, and a Griffiths phase, where $\chi(T)$ diverges as a power law with continuously varying exponents as $T \rightarrow 0$. The zigzag ladder, on the other hand, is topologically equivalent to single chains with both nearest neighbor (nn) and next nearest neighbor (nnn) interactions. Initial studies were confined to fairly small systems and concluded in favor of the existence of only one phase of the Griffiths type ${ }^{8}$ Subsequent investigations identified the presence of a small RS phase for weak nnn interactions ${ }^{10}$ Furthermore, the formation of large effective spins, with FM and AF nn and vanishing nnn couplings at the late stages of renormalization were taken as an indication of a LS phase. 10

The main purpose of this work is to establish in detail the effective low-energy equivalence between the random two-leg and zigzag ladders with the three random spin chains mentioned above. We do so by means of the RSRG method. In particular, we will show that at low energies the random two-leg ladder is equivalent to the dimerized AF spin- $1 / 2$ chain. In the course of this analysis, we will construct the full phase diagram of this model and confirm the existence of only two possible phases. Moreover, we will show that there are indeed two possible low-energy behaviors in the zigzag ladder. For small and weakly disordered nnn couplings, it is equivalent to a 
random AF spin-1/2 chain with its RS phase. If the nnn interactions are stronger, however, the system presents all the characteristic features of the LS phase of a random chain with both AF and FM couplings. We thus confirm the results of Ref. 10 by showing the same scaling of the effective cluster sizes and total spin values with energy as seen in those systems. However, the zigzag ladders can never exhibit the universal behavior observed in those systems at weak disorder. This is demonstrated by an exhaustive investigation of the dependence of the dynamical exponent $z$ on the shape and strength of the disorder distribution. Nevertheless, we do find different universal regions where $z$ remains fixed while the disorder is varied. The totality of our results thus serves to show that the universality classes of random spin ladders can already be found in simpler systems with nn interactions only.

The paper is organized as follows. In Sec. II we introduce the models we studied. The numerical results of the RSRG procedure of both the conventional two-leg and zigzag ladders are presented in Sec. III The unexpected behavior of the dynamical exponent of the zigzag ladders is explained in Sec. IV. We end with some final conclusions in Sec. $\mathrm{V}$ Appendix A discusses our method of calculation of the dynamical exponent $z$ and a brief discussion of the case of correlated disorder is given in Appendix B.

\section{THE MODEL}

We consider the following Hamiltonian, which describes an AF spin-1/2 chain with nearest neighbor (nn) and next nearest neighbor (nnn) interactions

$$
H=\sum_{i=1}^{N-1} J_{i} \mathbf{S}_{i} \cdot \mathbf{S}_{i+1}+\sum_{i=1}^{N-2} K_{i} \mathbf{S}_{i} \cdot \mathbf{S}_{i+2}
$$

where $\mathbf{S}_{i}$ is a spin-1/2 operator, $N$ is the total number of spins, and $J_{i}>0$ e $K_{i}>0$ are the nn and nnn random coupling constants, respectively. If $J_{i}=0$ for even $i$, this is the two-leg ladder Hamiltonian; if $J_{i}$ is in general nonzero, we have the zigzag ladder. The non-zero coupling constants $J_{i}$ and $K_{i}$ are in general independent random variables (see, however, Appendix (B). We take them to be respectively distributed in a power-law fashion (unless otherwise noted):

$$
\begin{gathered}
P_{J}(J)=\frac{1-\alpha}{J_{0}}\left(\frac{J_{0}}{J}\right)^{\alpha}, \quad 0<J<J_{0}, \\
P_{K}(K)=\frac{1-\alpha}{K_{0}}\left(\frac{K_{0}}{K}\right)^{\alpha} \quad, \quad 0<K<K_{0} .
\end{gathered}
$$

The exponent $\alpha(0 \leq \alpha<1)$ is a measure of the disorder strength and the ratio of cutoffs $\Lambda=K_{0} / J_{0}$ gives the typical relative strength between nnn and nn interactions.
In order to study these systems we employed the RSRG method introduced by Ma, Dasgupta and Hu. $\mathrm{I}^{1}$ Its decimation steps consist in isolating the strongest bond of the system $(\Omega)$, keeping only the lowest energy level of the bond, and renormalizing the remaining interactions by perturbation theory. The new renormalized coupling constants can be either ferromagnetic or antiferromagnetic. The details of the procedure have been extensively discussed in the published literature 5.8 .9 .10 and will be skipped here. As the largest energy scale is lowered from its initial value $\Omega_{0}=\max \left(J_{0}, K_{0}\right)$, an effective distribution of coupling constants is generated, which eventually flows towards a fixed point distribution. The low energy behavior of the system is governed by the remaining 'active' non-decimated spins at the scale of interest. 1.2 .5

When there are initially only nn interactions, further neighbor interactions are never generated.1.2.5 By contrast, in our case, the range of effective couplings rapidly increases as the RSRG is iterated (see Section IV for more details). However, as the fixed point is approached, interactions beyond nearest neighbors become extremely weak. If we then neglect interactions weaker than a certain upper bound $\left(\Omega_{\min } \approx 10^{-200} \Omega_{0}\right)^{11}$ the effective range actually extends only as far as the nearest neighbors. Thus, at the final stages of the RSRG, the ladders renormalize to effective nearest neighbor spin chains.

\section{NUMERICAL RESULTS}

In this section we show the numerical results obtained from the iteration of the RSRG for the two-leg and the zigzag ladders.

\section{A. Two-leg ladders}

We first focus on the two-leg ladders $\left(J_{2 i}=0\right)$. In our simulations we used chain lengths up to $N=200,000$.

In Fig. 11 we show the behavior of the fractions of nn, nnn, and 3rd nn bonds as functions of the energy scale $\Omega$. In addition, this Figure also shows the fraction of 'active' spins greater than $1 / 2$ and the fraction of antiferromagnetic coupling constants as functions of $\Omega$. The first thing to note is that the only significant couplings at the lowest energy scales $\left(\lesssim 10^{-3.5} \Omega_{0}\right)$ are nn couplings. Furthermore, these remaining couplings are all antiferromagnetic. This has been verified for all values of $\alpha$ and $\Lambda$.

Another important feature of the approach to the fixed point is the difference between the distributions of the odd bonds and the even ones. We have checked that, at the lowest energy scales, all the even bonds vanish while the odd ones are distributed according to

$$
P_{\text {Jodd }}(J) \sim J^{-1+1 / z},
$$

where $z$ is the dynamical exponent. 8.12 Thus, the system renormalizes to a collection of free effective random 


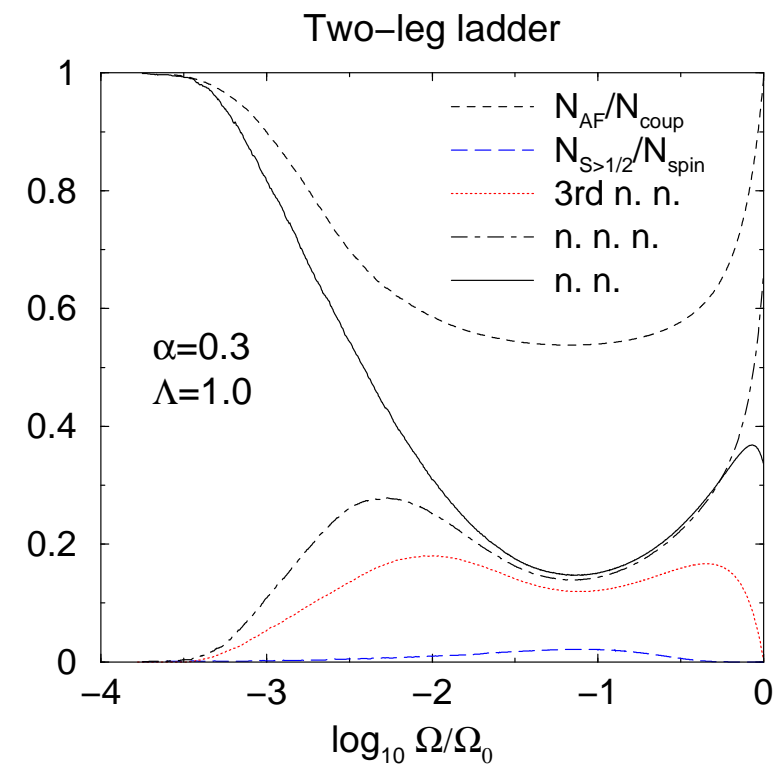

Figure 1: The behavior of the fractions of nearest-neighbor, next-nearest-neighbor, and third-nearest-neighbor coupling constants, the fraction of spins greater than $1 / 2$, and the fraction of antiferromagnetic couplings as functions of the energy scale $\Omega$. The initial number of spins is $N=200,000$, the disorder strength is $\alpha=0.3$, and the ratio $\Lambda=1.0$. The RSRG is iterated until only nearest-neighbor couplings are left. The data are averaged over 100 samples and the relative error is less than $2 \%$.

dimers. The average magnetic susceptibility is then given $\mathrm{by}^{2.4}$

$$
\chi \sim T^{1-z}
$$

If $z<1$, the density of states is suppressed at low energies and the system has a 'soft gap' (or a pseudo-gap). This is a remnant of the Haldane-type gap 13 of the clean two-leg ladder ${ }^{14}$ We therefore call it a disordered Haldane phase. Otherwise, the system shows no such suppression and is in a gapless (Griffiths) phase ${ }^{-8}$ We have determined the value of $z$, for different disorder strengths (see the Appendix $\mathrm{A}$ for details on the method of computation of $z$ ). As shown in Fig. 2 for $\Lambda=1$, a transition between these two phases occurs when $\alpha \approx 0.3$. In both phases the system is strongly dimerized. Within the length scale of the size of the effective dimers, the correlations decay as a power law. We believe this decay is similar to the case of random Heisenberg chains $\left(\sim 1 / r^{2}\right)$, since there are equal contributions coming from even and odd bond decimations. At larger length scales, the correlations are strongly (exponentially) suppressed. This overall behavior is apparent in Fig. 5 of Ref. 9. The crossover between the two regimes is governed by the effective dimer sizes, which are primarily determined by $\Lambda$ not $\alpha$.

Calculating the dynamical exponent $z$ for various values of $\Lambda$ and $\alpha$, one can construct the phase diagram of the two-leg ladder (Fig. 3). In the transition line, the dy-

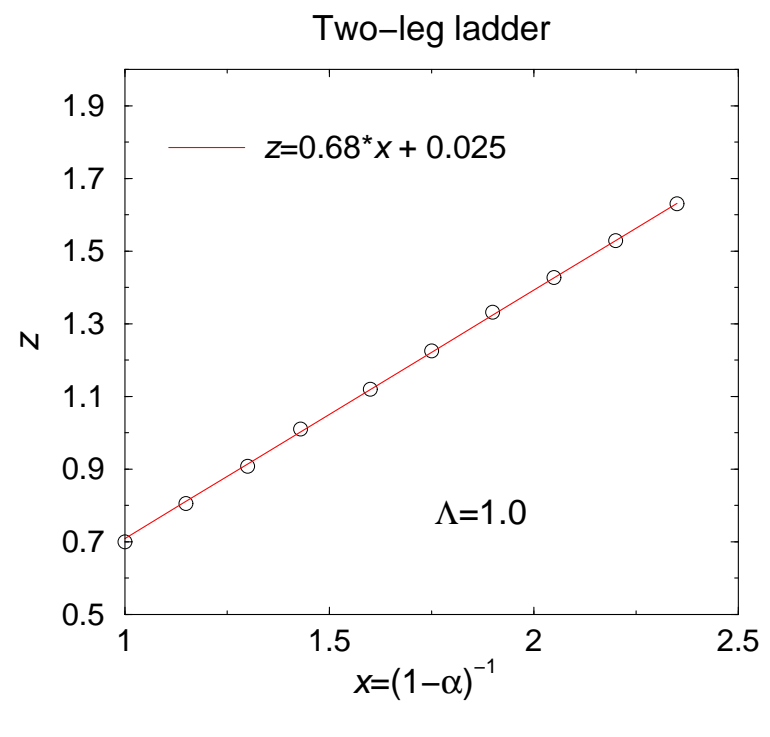

Figure 2: Variation of the dynamical exponent $z$ with the disorder strength $\alpha$ for $\Lambda=1.0 . \quad N=200,000$ is the initial number of spins in the ladder and the RSRG is iterated until there are only nearest-neighbor bonds left. The data are average over 5 samples and the relative error is about $3 \%$, comparable to the symbol size.

namical exponent equals one and the low-energy density of states and the magnetic susceptibility as $T \rightarrow 0$ are both constant.

From the inset of Fig. 3. we note that for $\Lambda \lesssim 10^{-2}$ the system is always in the Griffiths phase. We can understand this in the following manner. In the limit of $\Lambda \rightarrow 0$, the system reduces to a collection of disconnected dimers (the 'rungs' of the ladder), whose couplings are distributed according to Eq. (2a). Therefore, $z=(1-\alpha)^{-1} \geq 1$ and the system is always in a Griffiths phase. For small $\Lambda$, this behavior is preserved. For $10^{-2}<\Lambda<1.53$, the Haldane-type gap of the clean system gives rise to a 'soft gap' upon the introduction of weak disorder. For large disorder, the Griffiths phase re-emerges, with a diverging non-universal magnetic susceptibility. For $\Lambda>1.53$, only the Griffiths phase exists. This behavior smoothly connects with the $\Lambda \rightarrow \infty$ limit of two disconnected random Heisenberg chains, which is governed by the infinite randomness fixed point. 2 Formally, this limit corresponds to $z \rightarrow \infty$. We note that for any value of $\Lambda$, there are only two types of phases. 8.9

We stress the high degree of similarity between the disordered two-leg ladder and the dimerized random antiferromagnetic chain,$\frac{4}{,}$ which also shows analogous phases to the ones discussed here. Indeed, in both cases the RSRG flow leads to a fixed point with nn antiferromagnetic interactions only but with different distributions for even and odd bonds. Thus, the two systems clearly belong to the same universality class. 


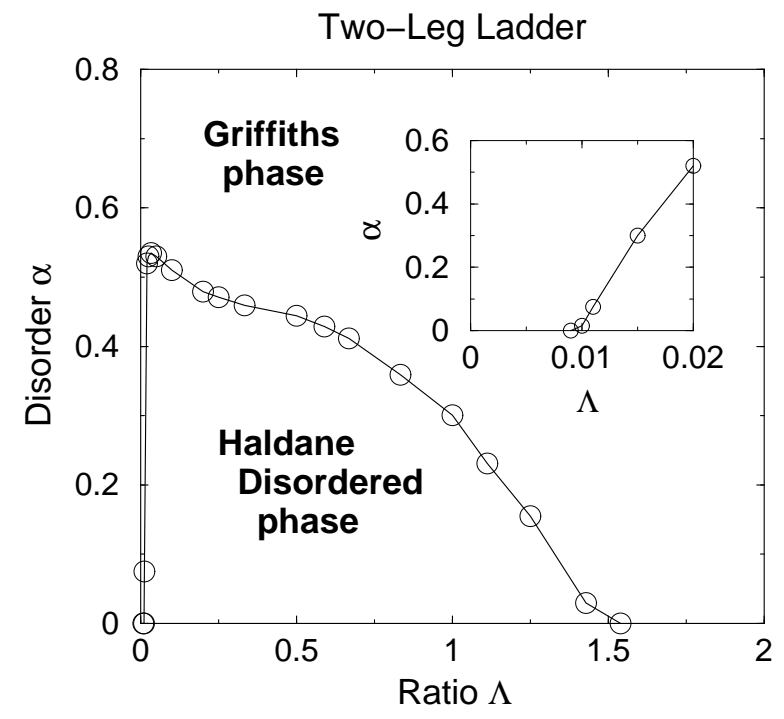

Figure 3: Phase diagram of the random two-leg ladder. The disordered Haldane phase is characterized by a 'soft gap' (or pseudo-gap), whereas in the Griffiths phase, the disorder completely destroys this remnant of the Haldane gap. In both phases the system is dimerized. The data error is about the size of the symbols.

\section{B. Zigzag ladders}

We now consider the zigzag ladders, with coupling constants distributed according to Eq. (2). We studied ladders with initial lengths of $N=640,000$.

Yusuf and Yang 10 have calculated the ratio of the average $\mathrm{nn}$ interactions to that of further neighbor interactions in zigzag ladders with correlated disorder. They have shown that this ratio increases tremendously as the energy scale is lowered. Indeed, if we plot the fraction of $\mathrm{nn}, \mathrm{nnn}$, and $3 \mathrm{rd} \mathrm{nn}$ interactions as functions of $\Omega$, for

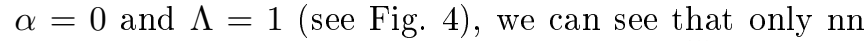
bonds survive at the lowest energies $\left(\approx 10^{-15} \Omega_{0}\right)$. This is similar to the case of two-leg ladders, as shown in Section IIIA and was verified in our simulations, irrespective of the values of $\alpha$ and $\Lambda$. However, in contrast to the two-leg ladder case, the nn bonds are not all antiferromagnetic. The asymptotic number of ferromagnetic and antiferromagnetic bonds is about the same, as shown also in Fig. 4. Finally, as also pointed out in Ref. 10, there is a rapid proliferation of spins greater than $1 / 2$. This is typical of the so-called Large Spin (LS) phase found in disordered Heisenberg chains with both FM and AF interactions. ${ }^{\frac{5}{3}}$ As we will see, this similarity is not fortuitous.

One of the distinguishing features of the disordered chains with both FM and AF interactions is the relation between the average spin size and the average cluster length. The average cluster size is related to the energy scale through the dynamical exponent $z$ by

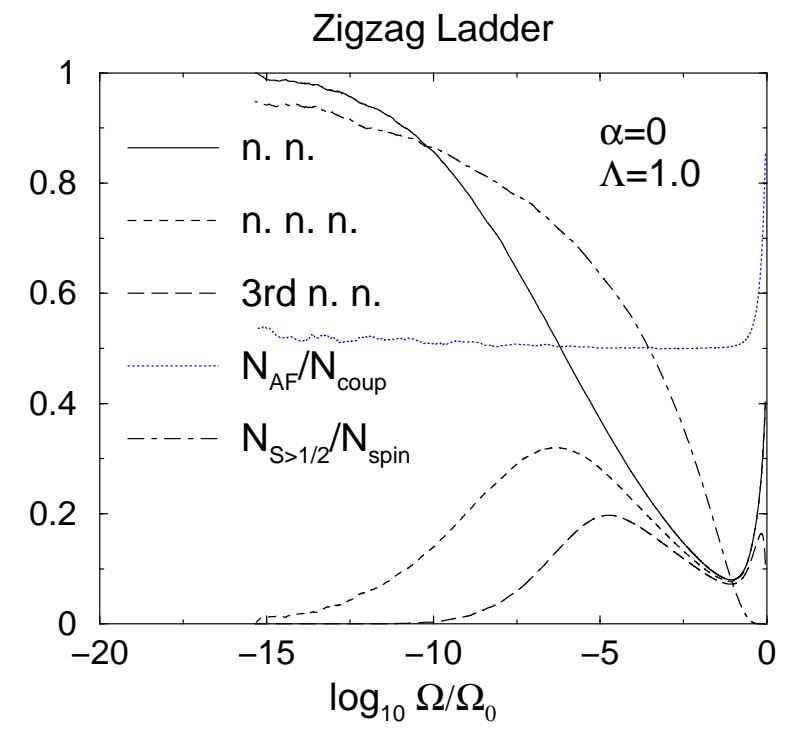

Figure 4: The fraction of nearest-neighbor, next-nearestneighbor, third-nearest-neighbor, the fraction of spins greater than $1 / 2$, and the fraction of AF couplings for zigzag ladders as functions of the energy scale $\Omega$. The initial number of spins is $N=640,000$, the disorder strength is $\alpha=0$ and the ratio $\Lambda=1.0$. The renormalization group is iterated until all the bonds in the system are between nearest-neighbors only. The data are averaged over 5 samples. For $\Omega / \Omega_{0} \geq 10^{-10}$ the data relative error is less than $2 \%$, otherwise, it is less than $8 \%$.

$<l>\sim \rho^{-1} \sim \Omega^{-1 / z}$ and the average spin size grows with the lowering energy scale according to $<S>\sim \Omega^{-\kappa}$. Both behaviors are observed in the zigzag ladders as shown in Fig. [5. As first pointed out by Westerberg et al.,$\frac{5}{,}$ the two exponents are related by $1 / z=2 \kappa$ in random chains with both FM and AF interactions. This follows from the fact that the main decimation process is not singlet formation but rather the formation of large spins from the random addition and subtraction of spin pairs. As a consequence, the cluster growth is characterized by a random walk in 'spin size space'. Again, we find this relation also holds for the zigzag ladders $(\kappa \approx 0.069$ and $1 / z \approx 0.14$ for the chain of Fig. 国). This is evidence that the zigzag ladders belong to the same universality class of the disordered chains with FM and AF interactions.

Our simulations also show that the fixed point distributions of FM and AF link excitation gaps are the same, with a characteristic power law dependence ${ }^{15}$

$$
P_{A F}(\Delta)=P_{F}(\Delta)=P(\Delta) \sim \Delta^{-1+1 / z},
$$

where $z$ is the same dynamical exponent obtained from the relation between $\langle l\rangle$ and $\Omega$ (see below for an explanation).

An interesting feature of the zigzag ladders is the following. In general, the total fixed point distribution of link excitation gaps is a linear combination of $\mathrm{AF}$ and 


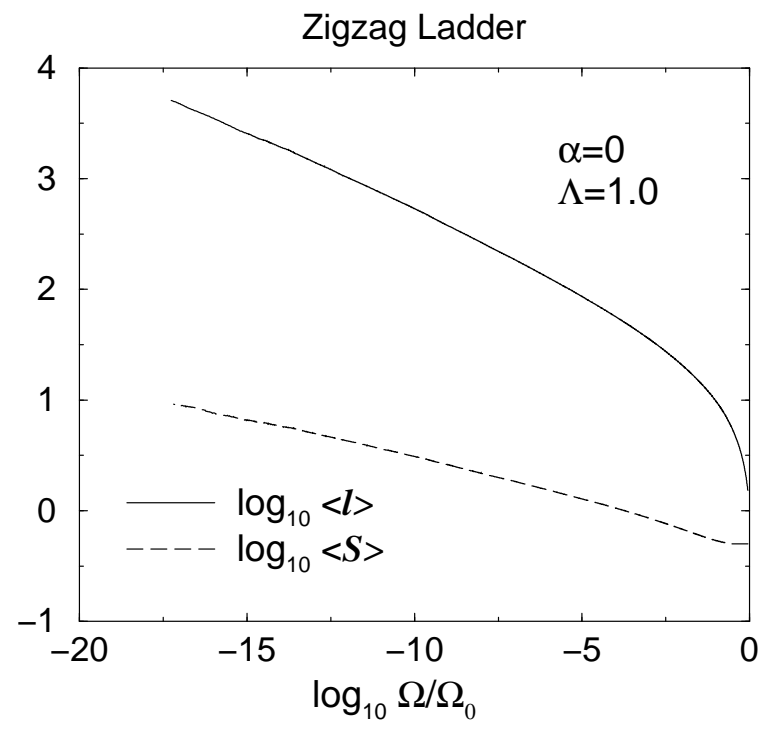

Figure 5: The average cluster size $\langle l\rangle$ and the average spin size $\langle S\rangle$ as functions of the energy scale $\Omega$. From a direct fit we get $\langle l\rangle \sim \Omega^{-0.14}$ and $\langle S\rangle \sim \Omega^{-0.069}$. The initial length of the system is $N=640,000, \alpha=0$ and $\Lambda=1.0$. The renormalization group is iterated until only nearest-neighbor couplings are left. The data were averaged over 5 samples and the sample to sample variation is less than $5 \%$.

FM contributions, namely,

$$
\begin{aligned}
P(\Delta)= & \frac{1}{\Omega}\left[\frac{x}{z_{A F}}\left(\frac{\Omega}{\Delta_{A F}}\right)^{1-1 / z_{A F}}\right. \\
& \left.+\frac{1-x}{z_{F M}}\left(\frac{\Omega}{\Delta_{F M}}\right)^{1-1 / z_{F M}}\right],
\end{aligned}
$$

where the first and second terms come from the $\mathrm{AF}$ and FM link distributions, respectively, and $x$ is the fraction of AF couplings. In our simulations, the fraction of AF bonds is $x \approx 0.53$ in the LS phase. The relation between length scale and the energy scale is given by ${ }^{2}$

$$
\frac{d \rho}{d \Omega}=P(\Delta=\Omega) \rho
$$

The coefficient in front of $P(\Delta=\Omega)$ is taken to be 1 because the main decimation process replaces 2 spins by 1. Solving Eq. (5) we get

$$
\rho \sim \Omega^{1 / z}
$$

with

$$
1 / z=x / z_{A F}+(1-x) / z_{F M}
$$

This general relation seems to have been unnoticed in previous studies. In particular, we note that weakly disordered chains with both $\mathrm{AF}$ and FM couplings have $1 / z_{F M} \approx 0.56,1 / z_{A F} \approx 0.30$, and $x \approx 0.63, \frac{5}{5}$ so that
$1 / z \approx 0.40$ which is reasonably close to the value of $0.44 \pm 0.02$ found directly in the simulations of Ref. 5 . In contrast, in the zigzag ladders, we have found $z_{A F}=$ $z_{F M}$, irrespective of the value of $x(x \approx 0.53$ in the LS phase). As a result, $z_{A F}=z_{F M}=z$.

Now, we would like to explore the disorder dependence of the dynamical exponent $z$. Fig. [6 illustrates the behavior of $z$ as a function of the disorder parameter $\alpha$ for various values of $\Lambda$. Note that the smallest value of $z$ is $1 / 0.15$. Westerberg et al. have found that for a range of weak disorder strengths, random chains with both FM and AF couplings are characterized by a universal value of $z_{U}=1 / 0.44^{\frac{5}{5}}$ Strongly singular disorder distributions, however, show non-universal disorderdependent values of $z$. As can be seen from Fig. 6] for initial power-law distributions of couplings, zigzag ladders always have $z>z_{U}$ and are never in the basin of attraction of the universal behavior.

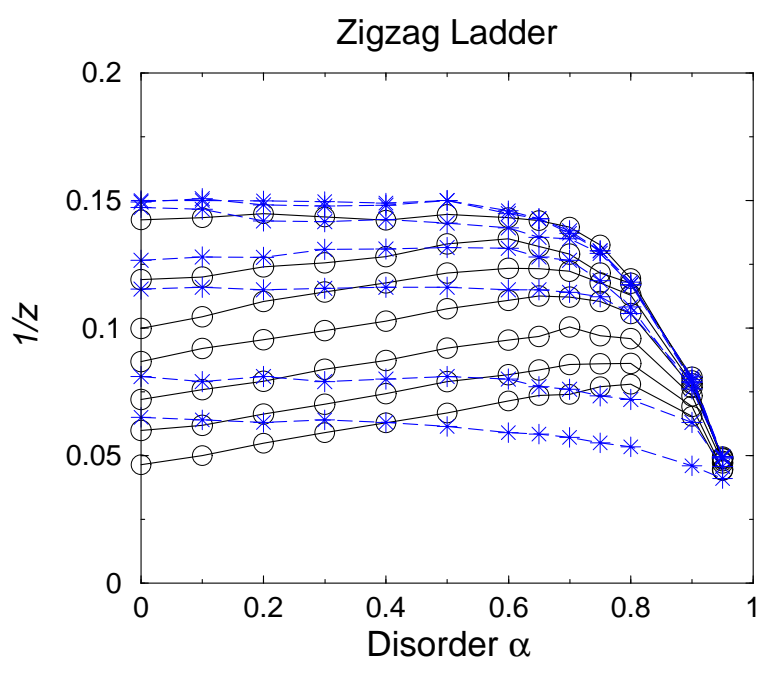

Figure 6: Variation of the dynamical exponent $z$ with the disorder parameter $\alpha$ for different values of $\Lambda$. From top to bottom, the solid lines with circles correspond to $\Lambda=1.0,0.6$, $0.4,0.2,0.1,0.05$, and 0.0025 , whereas the dashed lines with stars are for $\Lambda=0.8^{-1}, 0.6^{-1}, 0.4^{-1}, 0.2^{-1}, 0.1^{-1}, 10^{3}$, and $10^{6} . N=640,000$ is the initial number of spins in the ladder and the renormalization group is iterated until the bonds in the system are nearest-neighbor only. The data relative error is less than $10 \%$, about the size of the symbols.

For $\alpha \leq 0.6$ the behavior of $1 / z$ is linear in $\alpha$

$$
1 / z=a \alpha+b
$$

Note that, for $\Lambda \geq 1.0, a=0$ and for $1.0<\Lambda<1 / 0.4$ the system flows to a new universal fixed point where $1 / z=0.15 \pm 0.02$. This is similar to the random AF and FM chain ${ }^{\underline{5}}$ but the value of the dynamical exponents are different. We note from the general trend of the curves in Fig. [6] that the origin of this universal behavior seems to be the non-monotonic behavior of $z$ as one goes from $\Lambda \lesssim 1$ to $\Lambda \gtrsim 1$ (see also Fig. [7 below). This behavior, on 
the other hand, can be understood from simple physical arguments as shown below in Section IV

For $\Lambda \leq 0.4$ all the lines have the same slope $a=$ $0.039 \pm 0.001$ and the intercept $b$ varies in a logarithm manner with $\Lambda$, i.e., $b=b_{1}+b_{2} \ln \Lambda$, where $b_{1}=$ $0.12 \pm 0.01$ and $b_{2}=0.020 \pm 0.001$. Thus, there is a phase transition at the value of $\alpha$ where the dynamical exponent diverges, $\alpha=\alpha_{c}(\Lambda)=-\left(b_{2} \ln \Lambda+b_{1}\right) / a$. For $\alpha \leq \alpha_{c}(\Lambda)$, the system is governed by an infinite randomness fixed point where the magnitude of the spins does not grow and the FM couplings vanish. Indeed, the presence of such a RS phase was observed by Yusuf and Yang ${ }^{10}$ by direct calculation of $z$. Hence, we conclude that the system presents two phases. In the greater part of phase diagram it is in the LS phase, with a new universal fixed point in the region $1.0<\Lambda<1 / 0.4$ and $\alpha<0.6$. In addition, there is a tiny region where the system is in a RS phase.

For $\alpha>0.6$, all the curves converge to the point $\alpha=0.95$ and $z=1 / 0.05$. Note in this connection that $\alpha=0.95$ corresponds to an initial distribution which is the same as the fixed point one if $z=1 / 0.05$, cf. Eqs. (2a) and (4). This is a reflection of the inability of the decimation procedure, which is dominated by first order perturbation theory steps, to generate distributions which are more singular than the initial one.

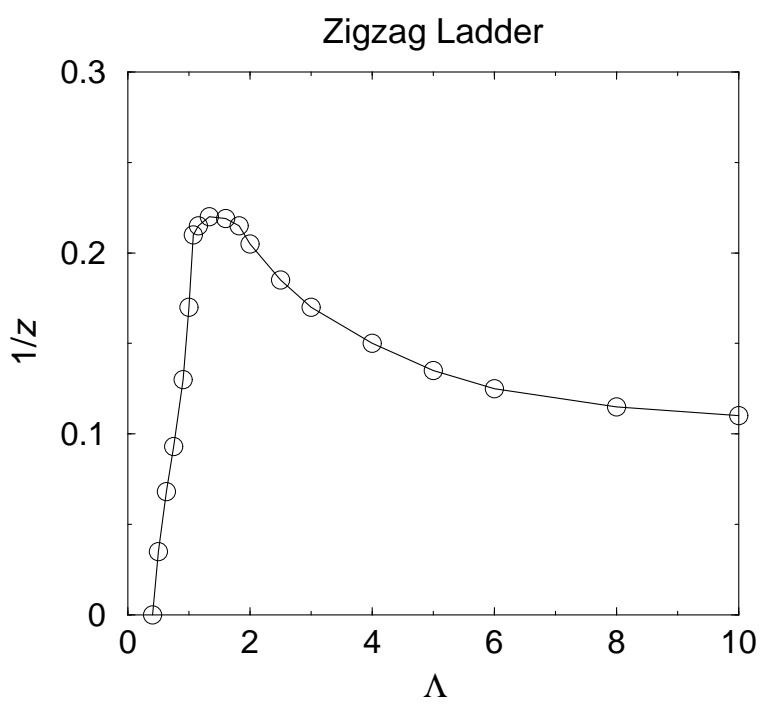

Figure 7: Variation of the dynamical exponent $z$ with the ratio $\Lambda$ for initial 'box distributions with a gap' ( $\delta=0.05$, see text for details). $N=200,000$ is the initial number of spins in the ladder and the renormalization group is iterated until the bonds in the system are nearest-neighbor only. The data relative error is less than $5 \%$, about the symbol size.

One might think that the universal behavior of disordered chains with $\mathrm{AF}$ and FM interactions where $z=z_{U}$ could be realized in zigzag ladders with disorder distributions which are not as singular as Eq. (2). This is not the case, however, as exemplified by the case of a 'box distribution with a gap', where the initial distributions are uniform and have support in $J_{0}-\delta<J<J_{0}$ and $K_{0}-\delta<K<K_{0}$ (we take $\max \left\{J_{0}, K_{0}\right\}=1$ ). We have explored several values of $\delta$ and found $z$ to be always greater than $z_{U}$. This is seen, for example, in the extreme case of $\delta=0.05$ in Fig. 7 where we plot $1 / z$ as a function of $\Lambda$. Note that there is a phase transition at $\Lambda=0.4$. To the left of this point, the system is in a RS spin- $1 / 2$ phase and the dynamical exponent diverges $z \sim-\ln \Omega$. For $\Lambda>0.4$, the system is in the LS phase. The smallest value of the dynamical exponent is $z=1 / 0.22$ (still greater than the universal $z_{U}$ found by Westerberg et. al) and for $\Lambda \gtrsim 10, z$ saturates at $z=1 / 0.11$. We conclude that the LS phase of the zigzag ladders is in the same universality class of random FM and AF chains with strong disorder but it does not reach the universal region found in those chains when the disorder is weak. It should be remembered that the clean system is gapless if $\Lambda \lesssim 0.24$ but spontaneously dimerized and gapful if $\Lambda \gtrsim 0.24{ }^{16}$ The topological nature of the dimerized state, however, makes it unstable with respect to any finite amount of disorder and the random system is always gapless $\underline{17}$

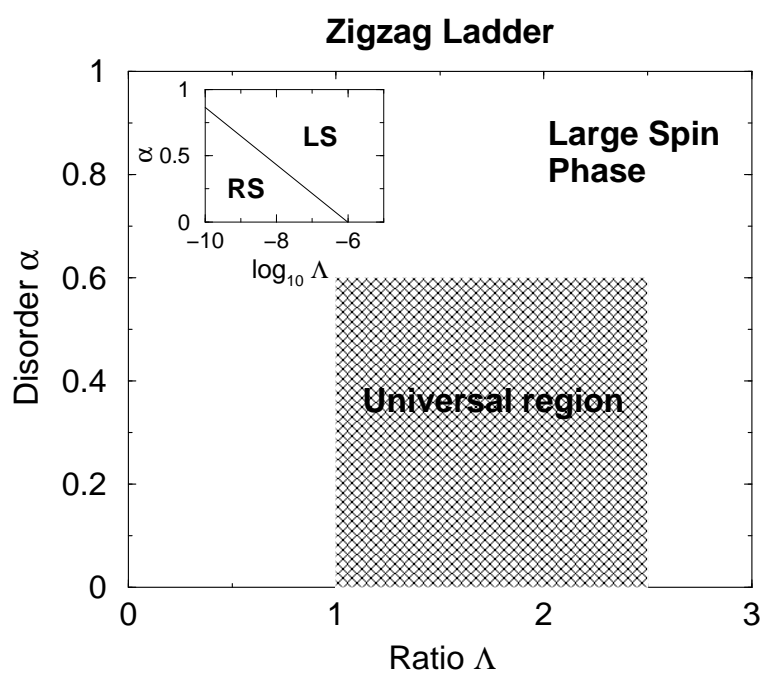

Figure 8: Phase diagram of the random zigzag ladder. The Large Spin phase dominates most of the parameter space. There is a universal region (the shaded area) for $1 \lesssim \Lambda \lesssim 2.5$ and $0 \lesssim \alpha \lesssim 0.6$ characterized by $z \approx 1 / 0.15$. There is also a tiny region for very small values of $\Lambda$ (see the inset), where the system is in a Random Singlet phase.

Based on the behavior of the dynamical exponent $z$, we can determine the phase diagram of the random zigzag ladder, as shown the Fig. 8. The Large Spin phase is dominant in most of the $(\alpha, \Lambda)$ parameter space. In this phase, the low energy physics of the system is governed by a fixed point where the mean size of the spin clusters grows when the energy scale is lowered, and they are weakly coupled. The distribution of link excitations gaps is not universal except in the shaded region. This 
universal region in the Large Spin phase is characterized by a dynamical exponent $z \approx 1 / 0.15$. The thermodynamic properties are well known ${ }^{5}$ the specific heat $C \sim T^{1 / z}|\ln T|$ and there is a Curie-like magnetic susceptibility $\chi \sim T^{-1}$. The average spin-spin correlation function decays in a logarithmic manner ${ }^{6}$ In addition to this phase, there is a tiny region where the system is in a RS phase (where $z \rightarrow \infty$, see the inset of the Fig. 8). Here, the low energy physics is governed by a universal infinite randomness fixed point. The specific heat $C \sim-1 / \ln ^{3} T$, the magnetic susceptibility $\chi \sim 1 /\left(T \ln ^{2} T\right)$, the mean correlation function $C_{m}(r) \sim r^{-2}$, and the typical correlation function $C_{t}(r) \sim \exp \left\{-r^{-1 / 2}\right\} \stackrel{2}{2}$ This phase was previously identified in Ref. 10, although its precise location differs somewhat from our results. We attribute this difference to finite-size effects and the different methods used for the characterization of RS behavior.

\section{ANOMALOUS DISORDER DEPENDENCE OF $z$ IN THE ZIGZAG LADDERS}

An intriguing aspect of the data shown in Fig. [6 is the fact that $z$ decreases as the initial disorder strength $\alpha$ is increased, for $\Lambda<1$ and $\alpha<0.6$ (solid lines of Figure 6). This behavior is unexpected since the weaker the initial disorder is, the stronger is the final effective one. As we will show, this anomalous behavior can be simply understood by analyzing the limiting behavior as $\Lambda \rightarrow 0$.

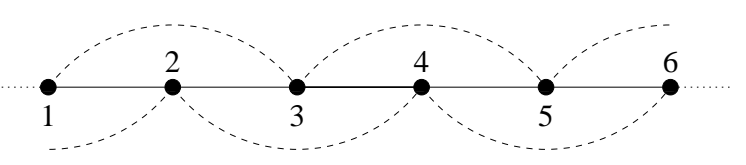

Figure 9: Schematic decimations at the earliest stages of the RG.

Let us start by writing down the effective couplings generated by the decimation procedure in its earliest stages when all the spins are spin- $1 / 2$ and the couplings are AF (see Fig. 9). Let us assume that the coupling between spins $S_{3}$ and $S_{4}$ is the largest one in the system, i.e., $\Omega=J_{34}$. After the decimation step, both spins are removed from the system because they are effectively frozen in a singlet state. The remaining spins are coupled with renormalized interactions as follows. The nn couplings are

$$
\begin{aligned}
& \tilde{J}_{12}=J_{12}-\frac{K_{13}\left(J_{23}-K_{24}\right)}{2 \Omega}, \\
& \tilde{J}_{25}=\frac{\left(J_{23}-K_{24}\right)\left(J_{45}-K_{35}\right)}{2 \Omega}, \\
& \tilde{J}_{56}=J_{56}-\frac{K_{46}\left(J_{45}-K_{35}\right)}{2 \Omega},
\end{aligned}
$$

the nnn ones are

$$
\begin{aligned}
& \tilde{K}_{15}=\frac{K_{13}\left(J_{45}-K_{35}\right)}{2 \Omega}, \\
& \tilde{K}_{26}=\frac{K_{46}\left(J_{23}-K_{24}\right)}{2 \Omega},
\end{aligned}
$$

and a third-nearest-neighbor coupling between spins $S_{1}$ and $S_{6}$ is generated

$$
\tilde{L}_{16}=\frac{K_{13} K_{46}}{2 \Omega} .
$$

First, we analyze the limit $\Lambda \ll 1$. In this case, it is easy to see that the renormalized nn, nnn, and 3rd nn couplings are of order $\mathcal{O}\left(\Lambda^{0}\right), \mathcal{O}\left(\Lambda^{1}\right)$, and $\mathcal{O}\left(\Lambda^{2}\right)$, respectively. Thus, if we neglect the $3 \mathrm{rd}$ nn coupling, the original form of the Hamiltonian is recovered, and the $\mathrm{nn}$ couplings remain stronger than the nnn ones. No FM coupling is generated and as the energy scale is lowered the nnn couplings vanish faster than the nn ones. The system flows to a random AF spin- $1 / 2$ chain in a RS phase. This scheme breaks down when a nn FM coupling appears leading to frustration. Among the three nn renormalized couplings, $\tilde{J}_{25}$ is the most likely to be FM with probability given by

$$
\begin{aligned}
P\left(\tilde{J}_{25}<0\right)= & 2 P\left(J_{23}<K_{24}\right) P\left(J_{45}>K_{35}\right) \\
= & 2\left\{\int_{0}^{\Lambda} P_{J}(J)\left[\int_{J}^{\Lambda} P_{K}(K) d K\right] d J\right\} \times \\
& \left\{1-\int_{0}^{\Lambda} P_{J}(J)\left[\int_{J}^{\Lambda} P_{K}(K) d K\right] d J\right\} \\
= & \Lambda^{1-\alpha}\left(1-\frac{1}{2} \Lambda^{1-\alpha}\right) .
\end{aligned}
$$

As expected, this probability is greater, the greater $\Lambda$ is, since $\Lambda$ governs the strength of frustration. But note also that it increases with $\alpha$. As more FM couplings are produced, the RG flow tends to deviate from the RS phase. As a result, the dynamical exponent will tend to be smaller. This explains the anomalous behavior we have found.

In the opposite limit $(\Lambda \gg 1)$, this picture no longer holds. Suppose that $\Omega=K_{35}$. In this case, the renormalized coupling between spins $S_{2}$ and $S_{4}$ is now a nn one

$$
\tilde{J}_{24}=K_{24}+\frac{J_{23}\left(J_{45}-J_{34}\right)}{2 \Omega}
$$


while the nnn coupling between $S_{1}$ and $S_{4}$ is

$$
\tilde{K}_{14}=\frac{K_{13}\left(J_{45}-J_{34}\right)}{2 \Omega} .
$$

It is clear that in this case the nn coupling tends to increase and the nnn one has a better chance of becoming a FM coupling in the earliest stages of the RG flow. This will rapidly enhance the frustration of the system and drive it away from the RS phase. In this limit, we expect the RS phase only at $\Lambda^{-1}=0$.

The competing tendencies at small and large $\Lambda$ lead to a minimum value of $z$ (Figs. [6] and [7). The presence of this minimum partially explains the universal region where $z$ is approximately constant at $\approx 6.7$ (Fig. [6).

A similar type of reasoning can be used to analyze the case of correlated disorder considered in Ref. 10. This is discussed in Appendix B

\section{CONCLUSIONS}

We have studied random AF two-leg and zigzag spin$1 / 2$ ladders using the real space renormalization group method of Ma, Dasgupta and Hu. We have determined the complete phase diagrams of these two general models in great detail by calculating the dynamical exponent $z$ and the range of low-energy effective interactions. The two-leg ladders show a gapped disordered Haldane region with a random dimer nature and a gapless Griffiths phase, but no random singlet phase. The zigzag ladder, on the other hand, can be either in a random singlet phase or a large spin phase.

One of our main findings is that throughout their phase diagrams these two models lead to effective low-energy models with nearest neighbor interactions only. This simplification had been noticed before in connection with the zigzag ladders $\stackrel{10}{\underline{10}}$ Our calculations confirm those results and generalize them to the two-leg ladders. Furthermore, by analyzing the structure of odd and even links in the case of the two-leg ladders and the scaling with energy of the large clusters of spins in the case of the zigzag ladders, we have been able to show the low-energy equivalence of these systems to the random dimerized AF spin chain and the random chain with AF and FM interactions, respectively. The low-energy equivalence between random systems with further neighbor yet short-ranged interactions to systems with nearest neighbor interactions only is likely to hold in general. However, there remains the possibility that one-dimensional models with longer ranged interactions are not decimated down to nearest neighbor spin chains in the process of the $\mathrm{RG}^{10}$ It would be interesting to determine the critical range beyond which such simplification does not occur.

While the zigzag ladder could be related to the random chain with both AF and FM chains, we have shown that their phase diagrams do not completely overlap. Indeed, the latter system has a universal fixed point at weak disorder (with $z \approx 2.27)^{5}$ which is inaccessible to the former. The zigzag ladder, on the other hand, has a region of parameter space with a new universal behavior: for initial distributions less singular than $P_{J}(x) \sim P_{K}(x) \sim x^{-\alpha_{c}}$, with $\alpha_{c} \approx 0.6$, and for $1.0 \lesssim \Lambda \lesssim 1 / 0.4$, the dynamical exponent is always $z \approx 6.7$. This critical disorder strength $\alpha_{c}$ is comparable to the one found in the random AF and FM chain, $\frac{5}{5}$ although we see no other similarities between these two regimes. We have been able to find a rough explanation for this universal behavior by ascribing it to competing tendencies which create a shallow valley where $z$ is approximately constant. It is possible that the universal regime of Ref. 5 has a similar origin.

\section{Acknowledgments}

We would like to acknowledge financial support from FAPESP through grants 03/00777-3 (JAH) and 01/00719-8 (EM) and CNPq 301222/97-5 (EM).

\section{Appendix A: THE CALCULATION OF THE DYNAMICAL EXPONENT $z$}

In this appendix, we would like to comment on our method of calculation of the dynamical exponent $z$. A common procedure, frequently used in the literature, is to decimate the system until one pair of spins is left. One can then calculate the excitation gap of this last dimer (the first gap $\Delta_{1}$ ). By repeating this procedure for different realizations of disorder one can obtain the distribution of first gaps. The dynamical exponent is obtained by fitting the behavior at small $\Delta_{1}$ to the power law in Eq. (3) $)^{8.18}$ This is shown in Fig. 10(a). Another option is to calculate the density of 'active' spins $\rho$ at the scale $\Omega$. The dynamical exponent $z$ is then obtained from its definition, through the relation between the length scale $l \sim \rho^{-1}$ and the energy scale $\Omega$

$$
\rho \sim \Omega^{1 / z} .
$$

This second method is exemplified in Fig. 10 (b), and the agreement is excellent. While the methods are equivalent it should be pointed out that the second method is computationally much faster, as a single realization of a large chain is sufficient for the determination of $z$. In our calculations, we have chosen to use the second method. This was actually crucial in the case of the zigzag ladders, where the asymptotic behavior can only be obtained with very large system sizes, $\frac{8}{\stackrel{8}{2}}$ rendering the first method very inefficient. This need for large sizes is due to the presence of long-range correlations in these systems ${ }^{6}$ as opposed to the exponential dependence of the two-leg ladders ${ }^{9}$ 

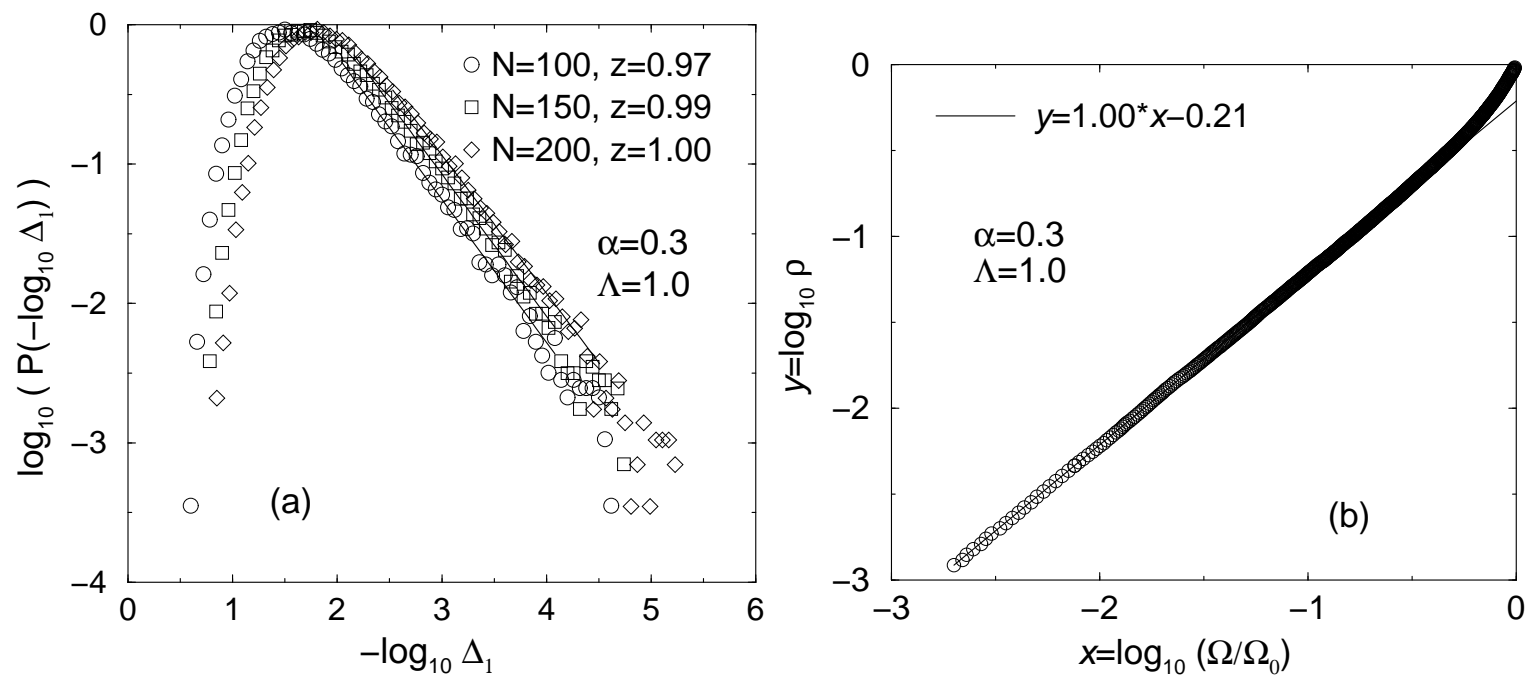

Figure 10: The calculation of the dynamical exponent $z$. (a) The distribution of first gaps $\Delta_{1}$ using 50,000 realizations for $N=100,150$, and 200, is fitted to $\log _{10} P\left(-\log _{10} \Delta_{1}\right)=$ const. $+(1 / z) \log \Delta_{1}$, and (b) $z$ is directly calculated from $\log _{10} \rho \sim$ const. $+(1 / z) \log _{10} \Omega$, where we used $N=200,000$. The data are from the decimation of a single chain. We verified that the variation with respect to other realizations of disorder is less than $1 \%$.

\section{Appendix B: ZIGZAG LADDERS WITH CORRELATED DISORDER}

Yusuf and Yang have also considered the case of zigzag ladders with correlations between the random $\mathrm{nn}$ and nnn couplings $\frac{10}{10}$ They have considered nn couplings distributed according to Eq. (2a) and nnn couplings given by

$$
K_{i}=\Lambda \frac{J_{i} J_{i+1}}{\Omega_{0}}
$$

Their analysis identified a RS phase for $\Lambda<0.5$, and a LS phase for $0.5<\Lambda<1.0$.

We can qualitatively analyze this case along the same lines as in Sec. IV Suppose $\Omega=J_{34}$ in Fig. 9 Using the above definition of $K_{i}$ in the earliest stages of the RG flow (when $\Omega \approx \Omega_{0}$ ), we have

$$
\tilde{J}_{25}=(1-\Lambda)^{2} \frac{J_{23} J_{45}}{2 \Omega},
$$

$$
\tilde{K}_{15}=\Lambda^{\prime} \frac{J_{12} \tilde{J}_{25}}{\Omega}
$$

$$
\tilde{K}_{26}=\Lambda^{\prime} \frac{\tilde{J}_{25} J_{56}}{\Omega}
$$

where $\Lambda^{\prime}=\Lambda /(1-\Lambda)$. Neglecting the 3 rd nn coupling and the renormalization of $J_{12}$ and $J_{56}$, the new Hamiltonian has the same form as the original one, except for the fact that the anisotropy parameter for $\tilde{K}_{15}$ and $\tilde{K}_{26}$ has been renormalized. In the first RG decimations, no FM couplings can arise. However, in a second run there is a finite probability for FM couplings to appear if $\Lambda^{\prime}>1$. This happens if $\Lambda>0.5$. The presence of FM couplings introduces frustration which is the mechanism that drives the system away from the RS phase towards the LS phase.
* Electronic address: joseabel@ifi.unicamp.br

† Electronic address: emiranda@ifi.unicamp.br

1 S.-k. Ma, C. Dasgupta, and C.-k. Hu, Phys. Rev. Lett. 43, 1434 (1979); C. Dasgupta and S.-k. Ma, Phys. Rev. B 22, 1305 (1980).

2 D. S. Fisher, Phys. Rev. B 50, 3799 (1994).

3 C. A. Doty and D. S. Fisher, Phys. Rev. B 45, 2167 (1992).

${ }^{4}$ R. A. Hyman, K. Yang, R. N. Bhatt, and S. M. Girvin Phys. Rev. Lett. 76, 839 (1996).
5 E. Westerberg, A. Furusaki, M. Sigrist, and P. A. Lee, Phys. Rev. B 55, 12578 (1997).

6 T. Hikihara, A. Furusaki, and M. Sigrist, Phys. Rev. B 60, 12116 (1999).

7 For a review, see E. Dagotto and T. M. Rice, Science 271, 618 (1996).

${ }^{8}$ R. Mélin, Y.-C. Lin, P. Lajkó, H. Rieger, and F. Iglói, Phys. Rev. B 65, 104415 (2002).

${ }^{9}$ E. Yusuf and K. Yang, Phys. Rev. B 65, 224428 (2002). 
10 E. Yusuf and K. Yang, Phys. Rev. B 68, 024425 (2003).

11 We take $\Omega_{\min }$ to be well below the lowest energy scale down to which we decimate $\left(\approx 10^{-40} \Omega_{0}\right)$, so that the neglected couplings are utterly irrelevant. We have checked that the results do not change if we use other values of $\Omega_{\min }$ consistent with this constraint.

12 D. S. Fisher, Phys. Rev. Lett. 69, 534 (1992); D. S. Fisher, Phys. Rev. B 51, 6411 (1995).

13 F. D. M. Haldane, Phys. Lett. 93A, 464 (1983).

14 E. Dagotto, J. Riera, and D. J. Scalapino, Phys. Rev. B. 45, 5744 (1992); G. Sierra, J. Phys. A 29, 3299 (1996).
15 We follow the convention of Ref. 5 and define $\Delta$ as the energy difference between the ground and first excited multiplets of a given pair of coupled spins.

16 F. D. M. Haldane, Phys. Rev. B 25, 4925 (1982); K. Okamoto and K. Nomura, Phys. Lett. A 169, 433 (1992); S. Eggert, Phys. Rev. B 54, 9612 (1996).

17 K. Yang, R. A. Hyman, R. N. Bhatt, and S. M. Girvin, J. Appl. Phys. 79, 5096 (1996).

18 A. Saguia, B. Boechat, and M. A. Continentino, Phys. Rev. Lett. 89, 117202 (2002). 